\title{
COMMON FIXED POINT RESULTS IN RANDOM FUZZY METRIC SPACE
}

\author{
Manoj Shukla ${ }^{1}$, R. P. Dubey ${ }^{2}$ and Shailendra Kumar Patel ${ }^{3}$ \\ ${ }^{1}$ Govt. Model Science College (autonomous), Jabalpur(M.P.) India \\ ${ }^{2}$ Dr C.V. Raman Institude of Sci. and Techn. Kota Bilaspur (C.G) India \\ ${ }^{3}$ Bhilai Institute of Tech., Raipur (C.G) India
}

Keywords: Random Fuzzy Metric Space, weak commuting mappings, rational expression, Common Fixed Points.

\begin{abstract}
In this paper we have proved some fixed point theorems in random fuzzy metric space for three mappings which satisfying some contractions. Also here we consider rational expression in contraction.
\end{abstract}

\section{INTRODUCTION AND PRILIMINARIES}

Probabilistic functional analysis has emerged as one of the important mathematical disciplines in view of its role in analyzing probabilistic models in the applied sciences. The study of fixed points of random operators forms a central topic in this area. The Prague school of probabilistic initiated its study in the 1950. However, the research in this area flourished after the publication of the survey article of Bharucha-Reid [3]. Since then many interesting random fixed point results and several applications have appeared in the literature; for example the work of Beg and Shahazad $[1,2]$.

In recent years, the study of random fixed points have attracted much attention some of the recent literatures in random fixed points may be noted in [1,2,3,5,6,7].In particular ,random iteration schemes leading to random fixed point of random operators have been discussed in $[5,6,7]$. Also, so many works on fuzzy metric space have been done by $[8,9,10]$. Here we are going to prove some problems in random fuzzy metric space.

We require some definitions and results to prove our results.

Definition 1. A binary operation $*:[0,1] \times[0,1] \rightarrow[0,1]$ is a continuous t-norm if it satisfies the following conditions

(1) * is associative and commutative,

(2) $*$ is continuous,

(3) $\mathrm{a}^{*} 1=\mathrm{a}$ for all $\mathrm{a} \in[0,1]$,

(4) $\mathrm{a}^{*} \mathrm{~b} \leq \mathrm{c} * \mathrm{~d}$ whenever $\mathrm{a} \leq \mathrm{c}$ and $\mathrm{b} \leq \mathrm{d}$, for each $\mathrm{a}, \mathrm{b}, \mathrm{c}, \mathrm{d} \in[0,1]$.

Two typical examples of continuous t-norm are

$a^{*} b=a b$ and $a^{*} b=\min (a, b)$.

Definition 2. Let $(\Omega, \Sigma)$ be a measurable space with $\Sigma$ a sigma algebra of subsets of $\Omega$ and $M$ a non-empty subset of a metric space $X=(X, d)$. Let $2 \mathrm{M}$ be the family of all non-empty subsets of $M$ and $C(M)$ the family of all nonempty closed subsets of $M$. A mapping G: $\Omega \rightarrow 2 \mathrm{M}$ is called measurable if, for each open subset $U$ of $M, G^{-1}(U) \in \Sigma$, where $G^{-1}(U)=\{w \in \Omega: G(w) \cap U \neq \phi\}$.

Definition 3. A mapping $\xi$ : $\Omega \rightarrow M$ is called a measurable selector of a measurable mapping $\mathrm{G}: \Omega$ $\rightarrow 2^{M}$ if $\xi$ is measurable and $\xi(w) \in G(w)$ for each $w \in \Omega$.

Definition 4. A mapping $\mathrm{T}: \Omega \times \mathrm{M} \rightarrow \mathrm{X}$ is said to be a random operator if, for each fixed $\mathrm{x} \in \mathrm{M}$, $\mathrm{T}(., \mathrm{x}): \Omega \rightarrow \mathrm{X}$ is measurable. 
Definition 5. A measurable mapping $\xi: \Omega \rightarrow \mathrm{M}$ is a random fixed point of a random operator $\mathrm{T}: \Omega$ $\times \mathrm{M} \rightarrow \mathrm{X}$ if $\xi(w)=\mathrm{T}(w, \xi(w))$ for each

Definition 6. Let $(\Omega, \Sigma)$ be a measurable space with $\Sigma$ a sigma algebra of subsets of $\Omega$. The 3tuple $\left(X, M,{ }^{*}\right)$ is called a Random Fuzzy Metric Space (RFM space) if $\mathrm{X}$ is an arbitrary (non empty) set and $\mathrm{C}$ is a non empty subset of $\mathrm{X}=(\mathrm{X}, \mathrm{d})$ also * is a continuous t-norm and $\mathrm{M}$ is a fuzzy set in $(\Omega \mathrm{xCx}(\Omega \mathrm{xCx}(\Omega \mathrm{x}[0, \infty))$ satisfying the following conditions for all $\mathrm{x}(\xi), \mathrm{y}(\xi)$ in $(\Omega \mathrm{xCx})$,

(1) $\mathrm{M}(\mathrm{x}(\xi), \mathrm{y}(\xi), 0(\xi))=0$,

(2) $M(\mathrm{x}(\xi), \mathrm{y}(\xi), \mathrm{t}(\xi))=1$ for all $\mathrm{t}(\xi)>0$ if and only if $\mathrm{x}(\xi)=\mathrm{y}(\xi)$,

(3) $\quad M(\mathrm{x}(\xi), \mathrm{y}(\xi), \mathrm{t}(\xi))=M(\mathrm{y}(\xi), \mathrm{x}(\xi), \mathrm{t}(\xi))$,

(4) $\quad M(\mathrm{x}(\xi), \mathrm{y}(\xi), \mathrm{t}(\xi)) * M(\mathrm{x}(\xi), \mathrm{z}(\xi), \mathrm{s}(\xi)) \leq M(\mathrm{x}(\xi), \mathrm{z}(\xi), \mathrm{t}(\xi)+\mathrm{s}(\xi))$,

(5) $\quad M(\mathrm{x}(\xi), \mathrm{y}(\xi), \alpha(\xi):[0,1] \rightarrow[0,1]$ is left continuous.

Note that $\mathrm{M}(\mathrm{x}(\xi), \mathrm{y}(\xi), \mathrm{t}(\xi))$ can be thought as degree of nearness between $(x(\xi)$ and $y(\xi)$ with respect to $t(\xi)$

We identify $\mathrm{x}(\xi)=\mathrm{y}(\xi)$ with $\mathrm{M}(\mathrm{x}(\xi), \mathrm{y}(\xi), \mathrm{t}(\xi))=1$ for all measurable $\mathrm{t}(\xi)>0$ and $\mathrm{M}(\mathrm{x}(\xi)$, $\mathrm{y}(\xi), \mathrm{t}(\xi))=0$ with $\infty$. In the following example, we know that every metric induces a fuzzy metric.

Example (1) Let $(X, d)$ be a metric space.

Define $\mathrm{a} * \mathrm{~b}=\mathrm{a} \mathrm{b}$, or $\mathrm{a} * \mathrm{~b}=\min \{\mathrm{a}, \mathrm{b}\})$ and $\forall \mathrm{x}(\xi), \mathrm{y}(\xi) \in \Omega \mathrm{xC}$ and $\mathrm{t}(\xi)>0$,

$$
M(x(\xi), y(\xi), t(\xi))=\frac{t(\xi)}{t(\xi)+d(x(\xi), y(\xi))}
$$

Then $\left(\mathrm{X}, \mathrm{M},{ }^{*}\right)$ is a fuzzy metric space. We call this fuzzy metric $\mathrm{M}$ induced by the metric $\mathrm{d}$ the standard fuzzy metric.

Definition (7): Let (X, M,*) is a random fuzzy metric space.

(i) A sequence $\{\mathrm{xn}(\xi)\}$ in $\mathrm{X}$ is said to be convergent to a point $\mathrm{x} \in \mathrm{X}$,

$\lim _{n \rightarrow \infty} M\left(x_{n}(\xi), x(\xi), t(\xi)\right)=1$

(ii) A sequence $\left\{\mathrm{x}_{\mathrm{n}}(\xi)\right\}$ in $\mathrm{X}$ is called a Cauchy sequence if

$\lim _{n \rightarrow \infty} M\left(x_{n+p}(\xi), x_{n}(\xi), t(\xi)\right)=1, \forall t(\xi) \succ 0$ and $p \succ 0$

(iii) A random fuzzy metric space in which every Cauchy sequence is convergent is said to be complete.

Let $\left(\mathrm{X} . \mathrm{M},{ }^{*}\right)$ is a random fuzzy metric space with the following condition.

(FM-6) $\lim _{t \rightarrow \infty} M(x(\xi), y(\xi), t(\xi))=1, \forall x, y \in X$

Definition (8): A function $M$ is continuous in random fuzzy metric space iff whenever $x_{n}(\xi) \rightarrow x(\xi), y_{n}(\xi) \rightarrow y(\xi) \Rightarrow \lim _{n \rightarrow \infty} M\left(x_{n}(\xi), y_{n}(\xi), t(\xi)\right) \rightarrow M(x(\xi), y(\xi), t(\xi))$

Definition (9): Two mappings A and S on random fuzzy metric space $\mathrm{X}$ are weakly commuting iff $\mathrm{M}(\operatorname{ASu}(\xi), \operatorname{SAu}(\xi), \mathrm{t}(\xi)) \geq \mathrm{M}(\operatorname{Au}(\xi), \operatorname{Su}(\xi), \mathrm{t}(\xi))$

Lemma (i) For all $\mathrm{x}(\xi), \mathrm{y}(\xi) \in \mathrm{X}, \mathrm{M}(\mathrm{x}(\xi), \mathrm{y}(\xi), \mathrm{t}(\xi))$ is non -decreasing.

Lemma (ii) Let $\{\mathrm{yn}\}(\xi)\}$ be a sequence in a random fuzzy metric space $\left(\mathrm{X}, \mathrm{M},{ }^{*}\right)$ with the condition (FM -6) If there exists a number $q \in(0,1)$ such that 
$M\left(y_{n+2}(\xi), y_{n+1}(\xi), q t(\xi)\right) \geq M\left(y_{n+1}(\xi), y_{n}(\xi), t(\xi)\right), \forall t(\xi) \succ 0$ and $n=1,2,3 \ldots \ldots$,

then $\left\{y_{n}(\xi)\right\}$ is a cauchy sequence in $X$.

Lemma (iii) [28] If, for all $\mathrm{x}(\xi), \mathrm{y}(\xi) \in \mathrm{X}, \mathrm{t}(\xi)>0$ and for a number $\mathrm{q} \in(0,1)$,

$M(x(\xi), y(\xi), q t(\xi)) \geq M(x(\xi), y(\xi), t(\xi))$, then $x(\xi)=y(\xi)$

Fisher proved the following theorem for three mappings in complete metric space:

THEOREM (2A): Let $S$ and $T$ be continuous mappings of a complete metric space (X, d) into itself. Then $\mathrm{S}$ and $\mathrm{T}$ have a common fixed point in $\mathrm{X}$ iff there exists a continuous mapping $\mathrm{A}$ of $\mathrm{X}$ into $\mathrm{S}(\mathrm{X}) \cap \mathrm{T}(\mathrm{X})$, which commutes with $\mathrm{S}$ and $\mathrm{T}$ and satisfy:

$\mathrm{d}(\mathrm{Ax}, \mathrm{Ay}) \geq \alpha \mathrm{d}(\mathrm{Sx}, \mathrm{Ty})$

for all $\mathrm{x}, \mathrm{y} \in \mathrm{X}$ and $0<\alpha<1$.

Then S, T and A have a unique common fixed point.

\section{Main results}

In the present paper we extend Theorem (2A) to fuzzy metric space.

THEOREM (1.1): Let (X, M, *) be a complete random fuzzy metric space with the condition (FM6) and let $S$ and $T$ be continuous mappings of $X$, then $S$ and $T$ have a common fixed point in $X$ ifthere exists continuous mappings $A$ of $X$ into $S(X) \cap T(X)$ which commute weakly with $S$ and $T$ and

$$
M(A(\xi, x(\xi)), A(\xi, y(\xi)), q \xi(t)) \geq \min \left\{\begin{array}{l}
M(T(\xi, y(\xi)), A(\xi, y(\xi)), t(\xi)), M(S(\xi, x(\xi)), A(\xi, x(\xi)), t(\xi)), \\
M(S(\xi, x(\xi)), T(\xi, y(\xi)), t(\xi)), M(A(\xi, x(\xi)), T(\xi, y(\xi)), t(\xi)), \\
M(S(\xi, x(\xi)), A(\xi, y(\xi)), t(\xi)),
\end{array}\right\}
$$

for all $\mathrm{x}(\xi), \mathrm{y}(\xi) \in \mathrm{X}, \mathrm{t}(\xi)>0$, and $0<\mathrm{q}<1$. Then $\mathrm{S}, \mathrm{T}$ and $\mathrm{A}$ have a unique common fixed point.

PROOF: We define a sequence $\left\{\mathrm{x}_{\mathrm{n}}(\xi)\right\}$ such that $\mathrm{A}\left(\xi, \mathrm{x}_{2 \mathrm{n}}(\xi)\right)=\mathrm{S}\left(\xi, \mathrm{x}_{2 \mathrm{n}-1}(\xi)\right)$ and $\mathrm{A}\left(\xi, \mathrm{x}_{2 \mathrm{n}-1}(\xi)\right)$ $=\mathrm{T}(\xi, \mathrm{x} 2 \mathrm{n},(\xi) \mathrm{n}=1,2,--\ldots \ldots$

We shall prove that $\{\mathrm{A}(\xi, \mathrm{xn}(\xi))\}$ is a Cauchy sequence.

$$
\begin{aligned}
& M\left(A\left(\xi, x_{2 n}(\xi)\right), A\left(\xi, x_{2 n+1}(\xi), q t(\xi)\right) \geq \min \left\{\begin{array}{l}
M\left(T\left(\xi, x_{2 n+1}(\xi)\right), A\left(\xi, x_{2 n+1}(\xi)\right), t(\xi)\right), \\
M\left(S\left(\xi, x_{2 n}(\xi)\right), A\left(\xi, x_{2 n}(\xi)\right), t(\xi)\right), \\
M\left(S\left(\xi, x_{2 n}(\xi)\right), T\left(\xi, x_{2 n+1}(\xi)\right), t(\xi)\right), \\
M\left(A\left(\xi, x_{2 n}(\xi)\right), T\left(\xi, x_{2 n+1}(\xi)\right), t(\xi)\right), \\
M\left(S\left(\xi, x_{2 n}(\xi)\right), A\left(\xi, x_{2 n+1}(\xi)\right), t(\xi)\right)
\end{array}\right\}\right. \\
& M\left(A\left(\xi, x_{2 n}(\xi)\right), A\left(\xi, x_{2 n+1}(\xi)\right), q t(\xi)\right) \geq \min \left\{\begin{array}{l}
M\left(A\left(\xi, x_{2 n}(\xi)\right), A\left(\xi, x_{2 n+1}(\xi), t(\xi)\right),\right. \\
M\left(A\left(\xi, x_{2 n+1}(\xi)\right), A\left(\xi, x_{2 n}(\xi), t(\xi)\right),\right. \\
\left.M\left(A\left(\xi, x_{2 n+1}(\xi)\right), A\left(\xi, x_{2 n}(\xi)\right), t(\xi)\right), A\left(\xi, x_{2 n}(\xi)\right), t(\xi)\right), \\
M\left(A\left(\xi, x_{2 n+1}(\xi)\right), A\left(\xi, x_{2 n+1}(\xi)\right), t(\xi)\right)
\end{array}\right\}
\end{aligned}
$$




$$
\begin{aligned}
& =\min \left\{\begin{array}{l}
M\left(A\left(\xi, x_{2 n}(\xi)\right), A\left(\xi, x_{2 n+1}(\xi), t(\xi)\right), M\left(A\left(\xi, x_{2 n+1}(\xi)\right), A\left(\xi, x_{2 n}(\xi)\right), t(\xi)\right),\right. \\
M\left(A\left(\xi, x_{2 n+1}(\xi)\right), A\left(\xi, x_{2 n}(\xi)\right), t(\xi)\right), 1,1
\end{array}\right\} \\
& \geq \min \left\{\begin{array}{l}
M\left(A\left(\xi, x_{2 n-1}(\xi)\right), A\left(\xi, x_{2 n}(\xi)\right), \frac{t(\xi)}{q}\right), M\left(A\left(\xi, x_{2 n}(\xi)\right), A\left(\xi, x_{2 n-1}(\xi)\right), \frac{t(\xi)}{q}\right), \\
M\left(A\left(\xi, x_{2 n}(\xi)\right), A\left(\xi, x_{2 n-1}(\xi)\right), \frac{t(\xi)}{q}\right), 1,1
\end{array}\right\}
\end{aligned}
$$

Therefore

$M\left(A\left(\xi, x_{2 n}(\xi)\right), A\left(\xi, x_{2 n+1}(\xi)\right), q t(\xi)\right) \geq M\left(A\left(\xi, x_{2 n-1}(\xi)\right), A\left(\xi, x_{2 n}(\xi), \frac{t(\xi)}{q}\right)\right.$

By induction

$$
M\left(A\left(\xi, x_{2 k}(\xi)\right), A\left(\xi, x_{2 m+1}(\xi)\right), q t(\xi)\right) \geq M\left(A\left(\xi, x_{2 m}(\xi)\right), A\left(\xi, x_{2 k-1}(\xi)\right), \frac{t(\xi)}{q}\right)
$$

For every $\mathrm{k}$ and $\mathrm{m}$ in $\mathrm{N}$, Further if $2 \mathrm{~m}+1>2 \mathrm{k}$, then

$$
\begin{gathered}
M\left(A\left(\xi, x_{2 k}(\xi)\right), A\left(\xi, x_{2 m+1}(\xi)\right), q t(\xi)\right) \geq M\left(A\left(\xi, x_{2 k-1}(\xi)\right), A\left(\xi, x_{2 m}(\xi)\right), \frac{t(\xi)}{q}\right) \\
\ldots \ldots \ldots \ldots \ldots \ldots . . . \\
\ldots M\left(A\left(\xi, x_{0}(\xi)\right), A\left(\xi, x_{2 m+1-2 k}(\xi), \frac{t(\xi)}{q^{2 k}}\right)\right.
\end{gathered}
$$

If $2 \mathrm{k}>2 \mathrm{~m}+1$, then

$$
\begin{gathered}
M\left(A\left(\xi, x_{2 k}(\xi)\right), A\left(\xi, x_{2 m+1}(\xi)\right), q t(\xi)\right) \geq M\left(A\left(\xi, x_{2 k-1}(\xi)\right), A\left(\xi, x_{2 m}(\xi)\right), \frac{t(\xi)}{q}\right) \\
\quad \ldots \ldots \ldots . . . \\
. . . .
\end{gathered}
$$

By simple induction with (1.1 b) and (1.1c) we have

$$
M\left(A\left(\xi, x_{n}(\xi)\right), A\left(\xi, x_{n+p}(\xi), q t(\xi)\right) \geq M\left(A\left(\xi, x_{0}(\xi,) A\left(\xi, x_{p}(\xi)\right), \frac{t(\xi)}{q^{n}}\right)\right. \text {. }\right.
$$

For $\mathrm{n}=2 \mathrm{k}, \mathrm{p}=2 \mathrm{~m}+1$ or $\mathrm{n}=2 \mathrm{k}+1, \mathrm{p}=2 \mathrm{~m}+1$ and by $(\mathrm{FM}-4)$

$$
\begin{gathered}
M\left(A\left(\xi, x_{n}(\xi)\right), A\left(\xi, x_{n+p}(\xi)\right), q t(\xi)\right) \geq M\left(A\left(\xi, x_{0}(\xi)\right), A\left(\xi, x_{1}(\xi)\right), \frac{t(\xi)}{2 q^{n}}\right) * \\
M\left(A\left(\xi, x_{1}(\xi)\right), A\left(\xi, x_{p}(\xi)\right), \frac{t(\xi)}{q^{n}}\right) .
\end{gathered}
$$

If $\mathrm{n}=2 \mathrm{k}, \mathrm{p}=2 \mathrm{~m}$ or $\mathrm{n}=2 \mathrm{k}+1, \mathrm{p}=2 \mathrm{~m}$

For every positive integer $\mathrm{p}$ and $\mathrm{n}$ in $\mathrm{N}$, by nothing that

$$
M\left(A\left(\xi, x_{0}(\xi)\right), A\left(\xi, x_{p}(\xi)\right), \frac{t(\xi)}{q^{n}}\right) . \rightarrow 1 \text { as } n \rightarrow \infty
$$


Thus $\left\{\mathrm{Ax}_{\mathrm{n}}\right\}$ is a Cauchy sequence. Since the space $\mathrm{X}$ is complete there exists $\mathrm{z} \in \mathrm{X}$, such that $\lim _{n \rightarrow \infty} A\left(\xi, x_{n}(\xi)\right)=\lim _{n \rightarrow \infty} S\left(\xi, x_{2 n-1}(\xi)\right)=\lim _{n \rightarrow \infty} T\left(\xi, x_{2 n}(\xi)=z(\xi)\right.$ which is measurable

It follows that $\mathrm{A}(\xi, \mathrm{x}(\xi))=\mathrm{S}(\xi, \mathrm{z}(\xi))=\mathrm{T}(\xi, \mathrm{z}(\xi))$ and Therefore

$M(A(\xi, z(\xi)), A(\xi, A(\xi, z(\xi))), q t(\xi)) \geq \min \left\{\begin{array}{l}M(T(\xi, A(\xi, z(\xi))), A(\xi, A(\xi, z(\xi))), t(\xi)), \\ M(S(\xi, z(\xi)), A(\xi, z(\xi)), t(\xi)), \\ M(S(\xi, z(\xi)), T(\xi, A(\xi, z(\xi))), t(\xi)), \\ M(A(\xi, z(\xi)), T(\xi, A(\xi, z(\xi))), t(\xi)), \\ M(S(\xi, z(\xi)), A(\xi, A(\xi, z(\xi))), t(\xi)),\end{array}\right\}$

$M(A(\xi, z(\xi)), A(\xi, A(\xi, z(\xi))), q t(\xi)) \geq M(S(\xi, z(\xi)), T(\xi, A(\xi, z(\xi))), t(\xi))$

$\geq M(S(\xi, z(\xi)), A(\xi, T(\xi, z(\xi))), t(\xi))$

$\geq M(A(\xi, z(\xi)), A(\xi, A(\xi, z(\xi))), t(\xi))----$

$\geq M\left(A(\xi, z(\xi)), A(\xi, A(\xi, z(\xi))), \frac{t(\xi)}{q^{n}}\right)$

Since $\lim _{n \rightarrow \infty} M\left(A(\xi, z(\xi)), A(\xi, A(\xi, z(\xi))), \frac{t(\xi)}{q^{n}}\right)=1$,

so $A(\xi, z(\xi))=A(\xi, A(\xi, z(\xi)))$.

Thus $\mathrm{z}$ is common fixed point of $\mathrm{A}, \mathrm{S}$ and $\mathrm{T}$.

For uniqueness, let $\mathrm{v}(\xi)(\mathrm{v}(\xi) \neq \mathrm{z}(\xi))$ be another common fixed point of $\mathrm{S}$, T and A.

By (1.1 a) we write

$M(A(\xi, z(\xi)), A(\xi, v(\xi)), q t(\xi)) \geq \min \left\{\begin{array}{l}M(T(\xi, v(\xi)), A(\xi, v(\xi)), t(\xi)), \\ M(S(\xi, z(\xi)), A(\xi, z(\xi)), t(\xi)), \\ M(S(\xi, z(\xi)), T(\xi, v(\xi)), t(\xi)), \\ M(A(\xi, z(\xi)), T(\xi, v(\xi)), t(\xi)), \\ M(S(\xi, z(\xi)), A(\xi, v(\xi)), t(\xi))\end{array}\right\}$

$M(A(\xi, z(\xi)), A(\xi, v(\xi)), q t(\xi)) \geq \min \left\{\begin{array}{l}M(v(\xi), v(\xi), t(\xi)), M(z(\xi), z(\xi), t(\xi)), \\ M(z(\xi), v(\xi), t(\xi)), M(z(\xi), v(\xi), t(\xi)), \\ M(z(\xi), v(\xi), t(\xi))\end{array}\right\}$

$M(A(\xi, z(\xi)), A(\xi, v(\xi)), q t(\xi)) \geq M(z(\xi), v(\xi), t(\xi))$

This implies that

$M(z(\xi), v(\xi), q t(\xi)) \geq M(z(\xi), v(\xi), t(\xi))$

Therefore by lemma iii, we write $\mathrm{z}(\xi)=\mathrm{v}(\xi)$.

This completes the proof of Theorem (1.1)

Now we prove a result for rational expression. 
THEOREM (1.2): Let (X, M, *) be a complete fuzzy metric space with the condition (FM-6) and let $S$ and $T$ be continuous mappings of $X$ in $X$, then $S$ and $T$ have a common fixed point in $X$ if there exists continuous mappings $A$ of $X$ into $S(X) \cap T(X)$ which commute weakly with $S$ and $T$ and

$$
M\left(A \left(\xi, x(\xi), A(\xi, y(\xi), q t(\xi)) \geq \min \left\{\begin{array}{l}
M(T(\xi, y(\xi)), A(\xi, y(\xi)), t(\xi)), \\
M(S(\xi, x(\xi)), A(\xi, x(\xi)), t(\xi)), \\
M(S(\xi, x(\xi)), T(\xi, y(\xi)), t(\xi)), \\
\frac{M(S(\xi, x(\xi)), T(\xi, y(\xi)), t(\xi))}{M(\xi, x(\xi)), T(\xi, y(\xi)), t(\xi))}, \\
\frac{M(T(\xi, y(\xi)), A(\xi, y(\xi)), t(\xi))}{M(S(\xi, x(\xi)), A(\xi, x(\xi)), t(\xi))}, \\
\frac{M(S(\xi, x(\xi)), A(\xi, x(\xi)), t(\xi))}{M(T(\xi, y(\xi)), A(\xi, y(\xi)), t(\xi))},
\end{array}\right\}\right.\right.
$$

for all $\mathrm{x}, \mathrm{y} \in \mathrm{X}, \mathrm{t}>0$, and $0<\mathrm{q}<1$. Then $\mathrm{S}, \mathrm{T}$ and $\mathrm{A}$ have a unique common fixed point.

PROOF: We define a sequence $\left\{\mathrm{x}_{\mathrm{n}}(\xi)\right\}$ such that

$\mathrm{A}\left(\xi, \mathrm{x}_{2 \mathrm{n}}(\xi)\right)=\mathrm{S}\left(\xi, \mathrm{x}_{2 \mathrm{n}-1}(\xi)\right)$ and $\mathrm{A}\left(\xi, \mathrm{x}_{2 \mathrm{n}-1}(\xi)\right)=\mathrm{T}\left(\xi, \mathrm{x}_{2 \mathrm{n}}(\xi)\right) \mathrm{n}=1,2,--$

We shall prove that $\left\{\mathrm{A}\left(\xi, \mathrm{x}_{\mathrm{n}}(\xi)\right\}\right.$ is a Cauchy sequence. By $(1.2 \mathrm{a})$, we have

$M\left(A\left(\xi, x_{2 n}(\xi)\right), A\left(\xi, x_{2 n+1}(\xi)\right), q t(\xi)\right) \geq \min \left\{\begin{array}{l}M\left(T\left(\xi, x_{2 n+1}(\xi)\right), A\left(\xi, x_{2 n+1}(\xi), t(\xi)\right)\right), \\ M\left(S\left(\xi, x_{2 n}(\xi)\right), A\left(\xi, x_{2 n}(\xi)\right), t(\xi)\right), \\ M\left(S\left(\xi, x_{2 n}(\xi)\right), T\left(\xi, x_{2 n+1}(\xi)\right), t(\xi)\right), \\ M\left(A\left(\xi, x_{2 n}(\xi)\right), T\left(\xi, x_{2 n+1}(\xi)\right), t(\xi)\right) \\ \frac{M\left(T\left(\xi, x_{2 n+1}(\xi)\right), A\left(\xi, x_{2 n+1}(\xi)\right), t(\xi)\right)}{M\left(S\left(\xi, x_{2 n}(\xi)\right), A\left(\xi, x_{2 n}(\xi)\right), t(\xi)\right)}, \\ \frac{M\left(S\left(\xi, x_{2 n}(\xi)\right), A\left(\xi, x_{2 n}(\xi)\right), t(\xi)\right)}{M\left(T\left(\xi, x_{2 n+1}(\xi)\right), A\left(\xi, x_{2 n+1}(\xi)\right), t(\xi)\right)}\end{array}\right.$,

$M\left(A\left(\xi, x_{2 n}(\xi)\right), A\left(\xi, x_{2 n+1}(\xi)\right), q t(\xi)\right) \geq \min \left\{\begin{array}{l}M\left(A\left(\xi, x_{2 n}(\xi)\right), A\left(\xi, x_{2 n+1}(\xi)\right), t(\xi)\right), \\ M\left(A\left(\xi, x_{2 n+1}(\xi)\right), A\left(\xi, x_{2 n}(\xi)\right), t(\xi)\right), \\ M\left(A\left(\xi, x_{2 n+1}(\xi)\right), A\left(\xi, x_{2 n}(\xi)\right), t(\xi)\right), \\ M\left(A\left(\xi, x_{2 n+1}(\xi)\right), A\left(\xi, x_{2 n}(\xi)\right), t(\xi)\right) \\ \frac{M\left(A\left(\xi, x_{2 n}(\xi)\right), A\left(\xi, x_{2 n+1}(\xi)\right), t(\xi)\right)}{M\left(A\left(\xi, x_{2 n+1}(\xi)\right), A\left(\xi, x_{2 n}(\xi)\right), t(\xi)\right)} \\ \frac{M\left(A\left(\xi, x_{2 n+1}(\xi)\right), A\left(\xi, x_{2 n}(\xi)\right), t(\xi)\right)}{M\left(A\left(\xi, x_{2 n}(\xi)\right), A\left(\xi, x_{2 n+1}(\xi)\right), t(\xi)\right)}\end{array}\right\}$ 


$$
\begin{aligned}
& =\min \left\{\begin{array}{l}
M\left(A\left(\xi, x_{2 n}(\xi)\right), A\left(\xi, x_{2 n+1}(\xi)\right), t(\xi)\right), \\
M\left(A\left(\xi, x_{2 n+1}(\xi)\right), A\left(\xi, x_{2 n}(\xi)\right), t(\xi)\right), \\
M\left(A\left(\xi, x_{2 n+1}(\xi)\right), A\left(\xi, x_{2 n}(\xi)\right), t(\xi)\right), 1,1,1
\end{array}\right\} \\
& \geq \min \left\{M\left(A\left(\xi, x_{2 n-1}(\xi)\right), A\left(\xi, x_{2 n}(\xi)\right), \frac{t(\xi)}{q}\right), M\left(A\left(\xi, x_{2 n}(\xi)\right), A\left(\xi, x_{2 n-1}(\xi)\right), \frac{t(\xi)}{q}\right)\right\} \\
& \Rightarrow M\left(A\left(\xi, x_{2 n}(\xi)\right), A\left(\xi, x_{2 n+1}(\xi)\right), q t(\xi)\right) \geq M\left(A\left(\xi, x_{2 n-1}(\xi)\right), A\left(\xi, x_{2 n}(\xi)\right), \frac{t(\xi)}{q}\right)
\end{aligned}
$$

By induction

$$
M\left(A\left(\xi, x_{2 k}(\xi)\right), A\left(\xi, x_{2 m+1}(\xi)\right), q t(\xi)\right) \geq M\left(A\left(\xi, x_{2 m}(\xi)\right), A\left(\xi, x_{2 k-1}(\xi)\right), \frac{t(\xi)}{q}\right)
$$

For every $\mathrm{k}$ and $\mathrm{m}$ in $\mathrm{N}$, Further if $2 \mathrm{~m}+1>2 \mathrm{k}$, then

$$
\begin{gathered}
M\left(A\left(\xi, x_{2 k}(\xi)\right), A\left(\xi, x_{2 m+1}(\xi)\right), q t(\xi)\right) \geq M\left(A\left(\xi, x_{2 k-1}(\xi)\right), A\left(\xi, x_{2 m}(\xi)\right), \frac{t(\xi)}{q}\right) \ldots \ldots . \\
\quad \ldots \ldots \ldots . . M\left(A\left(\xi, x_{0}(\xi)\right), A\left(\xi, x_{2 m+1-2 k}(\xi)\right), \frac{t(\xi)}{q^{2 k}}\right)
\end{gathered}
$$

If $2 \mathrm{k}>2 \mathrm{~m}+1$, then

$$
\begin{aligned}
& M\left(A\left(\xi, x_{2 k}(\xi)\right), A\left(\xi, x_{2 m+1}(\xi)\right), q t(\xi)\right) \geq M\left(A\left(\xi, x_{2 k-1}(\xi)\right), A\left(\xi, x_{2 m}(\xi)\right), \frac{t(\xi)}{q}\right) \ldots \ldots \ldots \\
& \quad \ldots \ldots \ldots . . . \\
& \left.. A\left(\xi, x_{2 k-(2 m+1)}(\xi)\right), A\left(\xi, x_{0}(\xi)\right), \frac{t(\xi)}{q^{2 m+1}}\right)
\end{aligned}
$$

By simple induction with (1.2b) and (1.2c), We have $M\left(A\left(\xi, x_{n}(\xi)\right), A\left(\xi, x_{n+p}(\xi)\right), q t(\xi)\right) \geq M\left(A\left(\xi, x_{0}(\xi)\right), A\left(\xi, x_{p}(\xi)\right), \frac{t(\xi)}{q^{n}}\right)$.

For $\mathrm{n}=2 \mathrm{k}, \mathrm{p}=2 \mathrm{~m}+1$ or $\mathrm{n}=2 \mathrm{k}+1, \mathrm{p}=2 \mathrm{~m}+1$ and by $($ FM- 4$)$

$$
\begin{aligned}
& M\left(A\left(\xi, x_{n}(\xi)\right), A\left(\xi, x_{n+p}(\xi)\right), q t(\xi)\right) \\
& \geq M\left(A\left(\xi, x_{0}(\xi)\right), A\left(\xi, x_{1}(\xi)\right), \frac{t(\xi)}{2 q^{n}}\right) * M\left(A\left(\xi, x_{1}(\xi)\right), A\left(\xi, x_{p}(\xi)\right), \frac{t(\xi)}{q^{n}}\right) .
\end{aligned}
$$

If $\mathrm{n}=2 \mathrm{k}, \mathrm{p}=2 \mathrm{~m}$ or $\mathrm{n}=2 \mathrm{k}+1, \mathrm{p}=2 \mathrm{~m}$

For every positive integer $\mathrm{p}$ and $\mathrm{n}$ in $\mathrm{N}$, by nothing that

$M\left(A\left(\xi, x_{0}(\xi)\right), A\left(\xi, x_{p}(\xi)\right), \frac{t(\xi)}{q^{n}}\right) \cdot \rightarrow 1$ as $n \rightarrow \infty$

Thus $\left\{A\left(\xi, x_{n}(\xi)\right)\right\}$ is a Cauchy sequence.

Since the space $\mathrm{X}$ is complete there exists $\mathrm{z} \in \mathrm{X}$, such that $\lim _{n \rightarrow \infty} A\left(\xi, x_{n}(\xi)\right)=\lim _{n \rightarrow \infty} S\left(\xi, x_{2 n-1}(\xi)\right)=\lim _{n \rightarrow \infty} T\left(\xi, x_{2 n}(\xi)\right)=z$

It follows that $A(\xi, x(\xi))=S(\xi, z(\xi))=T(\xi, z(\xi))$ and 
Therefore

$M\left(A\left(\xi, z(\xi), A(\xi, A(\xi, A(\xi, z(\xi))), q t(\xi)) \geq \min \left\{\begin{array}{l}M(T(\xi, A(\xi, z(\xi))), A(\xi, A(\xi, z(\xi))), t(\xi)), \\ M(S(\xi, z(\xi)), A(\xi, z(\xi)), t(\xi)), \\ M(S(\xi, z(\xi)), T(\xi, A(\xi, z(\xi))), t(\xi)), \\ \frac{M(S(\xi, z(\xi)), T(\xi, A(\xi, z(\xi))), t(\xi))}{M(A(\xi, z(\xi)), T(\xi, A(\xi, z(\xi))), t(\xi))} \\ \frac{M(T(\xi, A(\xi, z(\xi))), A(\xi, A(\xi, z(\xi))), t(\xi))}{M(S(\xi, z(\xi)), A(\xi, z(\xi)), t(\xi))}, \\ \frac{M(S(\xi, z(\xi)), A(\xi, z(\xi)), t(\xi))}{M(T(\xi, A(\xi, z(\xi)), A(\xi, A(\xi, z(\xi))), t(\xi))},\end{array}\right\}\right.\right.$

$M(A(\xi, z(\xi)), A(\xi, A(\xi, z(\xi))), q t(\xi)) \geq M(S(\xi, z(\xi)), T(\xi, A(\xi, z(\xi))), t(\xi))$

$\geq M(S(\xi, z(\xi)), A(\xi, T(\xi, z(\xi))), t(\xi)) \geq M(A(\xi, z(\xi)), A(\xi, A(\xi, z(\xi))), t(\xi))$ $\geq M\left(A(\xi, z(\xi)), A(\xi, A(\xi, z(\xi))), \frac{t(\xi)}{q^{n}}\right)$

Since $\lim _{n \rightarrow \infty} M\left(A(\xi, z(\xi)), A(\xi, A(\xi, z(\xi))), \frac{t(\xi)}{q^{n}}\right)=1$

$\Rightarrow A(\xi, z(\xi))=A(\xi, A(\xi, z(\xi)))$

Thus $\mathrm{z}(\xi)$ is common fixed point of $\mathrm{A}, \mathrm{S}$ and $\mathrm{T}$.

For uniqueness, let $\mathrm{v}((\xi)(\mathrm{v}(\xi) \neq \mathrm{z}(\xi))$ be another common fixed point of $\mathrm{S}$, T and A.

By (1.2a), we write

$M(A(\xi, z(\xi)), A(\xi, v(\xi)), q t(\xi)) \geq \min \left\{\begin{array}{l}M(T(\xi, v(\xi)), A(\xi, v(\xi)), t(\xi)), \\ M(S(\xi, z(\xi)), A(\xi, z(\xi)), t(\xi)), \\ M(S(\xi, z(\xi)), T(\xi, v(\xi)), t(\xi)), \\ M(A(\xi, z(\xi)), T(\xi, v(\xi)), t(\xi)) \\ \frac{M(T(\xi, v(\xi)), A(\xi, v(\xi)), t(\xi))}{M(S(\xi, z(\xi)), A(\xi, z(\xi)), t(\xi))}, \\ \frac{M(S(\xi, z(\xi)), A(\xi, z(\xi)), t(\xi))}{M(T(\xi, v(\xi)), A(\xi, v(\xi)), t(\xi))},\end{array}\right\}$

$M(A(\xi, z(\xi)), A(\xi, v(\xi)), q t(\xi)) \geq M(z(\xi), v(\xi), t(\xi))$

This implies that

$M(z(\xi), v(\xi), q t(\xi)) \geq M(z(\xi) v,(\xi), t(\xi))$

Therefore by lemma iii, we write $\mathrm{z}(\xi)=\mathrm{v}(\xi)$.

This completes the proof of Theorem (1.2).

Now we are proving another common fixed point theorem, which is motivated by Som [10]. 
THEOREM (1.3) Let $(\mathrm{X}, \mathrm{M}, *)$ be a complete Fuzzy Random metric space with the condition (FM-6) and let $F$ and $T$ be continuous mappings of $X$ in $X$. Let $A$ be a self mapping of $X$ satisfying $\{\mathrm{A}, \mathrm{F}\}$ and $\{\mathrm{A}, \mathrm{T}\}$ are $\mathrm{R}$-weakly commuting and

$$
\mathrm{A}(\mathrm{X}) \subseteq \mathrm{F}(\mathrm{X}) \cap \mathrm{T}(\mathrm{X})
$$

for all $\mathrm{x}(\xi), \mathrm{y}(\xi) \in \mathrm{X}$, Where $\mathrm{r}:[0,1] \rightarrow[0,1]$ is a continuous function such that $\mathrm{r}(\mathrm{t})>\mathrm{t}$, for each $\mathrm{o} \leq$ $\mathrm{t} \leq 1$ and $\mathrm{r}(\mathrm{t})=1$ for $\mathrm{t}=1$. The sequence $\{\mathrm{xn}(\xi)\}$ and $\{\mathrm{yn}(\xi)\}$ in $\mathrm{X}$ are such that

$$
\begin{aligned}
& x_{n}(\xi) \rightarrow x(\xi), y_{n}(\xi) \rightarrow y(\xi) \\
& \Rightarrow M\left(x_{n}(\xi), y_{n}(\xi), t(\xi)\right) \rightarrow M(x(\xi), y(\xi), t(\xi)), \\
& \text { where } t(\xi)>0 \text { is measurable. }
\end{aligned}
$$

Then $\mathrm{F}$, $\mathrm{T}$ and $\mathrm{A}$ have a unique common fixed point in $\mathrm{X}$.

PROOF: We define a sequence $\left\{\mathrm{x}_{\mathrm{n}}(\xi)\right\}$ such that

$\operatorname{Fx}_{2 \mathrm{n}+1}(\xi)=\mathrm{Ax}_{2 \mathrm{n}}(\xi)$ and $\operatorname{Tx}_{2 \mathrm{n}+2}(\xi)=\mathrm{Ax}_{2 \mathrm{n}+1},(\xi), \mathrm{n}=1,2, ., ., \ldots$

We shall prove that $\left\{A_{x n}(\xi)\right\}$ is a Cauchy sequence for $n=0,1,2----$

$G_{n}(\xi)=M\left(A x_{n}(\xi), A x_{n+1}(\xi), t(\xi)\right)<1 ; n=0,1,2,3--$

$G_{2 n}(\xi)=M\left(A x_{2 n+1}(\xi), A x_{2 n}(\xi), t(\xi)\right)$

$$
\geq r\left[\operatorname{Min}\left\{\begin{array}{l}
M\left(F\left(\xi, x_{2 n+1}(\xi)\right), T\left(\xi, x_{2 n}(\xi)\right), t(\xi)\right), M\left(F\left(\xi, x_{2 n+1}(\xi)\right), A\left(\xi, x_{2 n+1}(\xi)\right), t(\xi)\right), \\
M\left(F\left(\xi, x_{2 n+1}(\xi)\right), A\left(\xi, x_{2 n}(\xi)\right), t(\xi)\right), M\left(T\left(\xi, x_{2 n}(\xi)\right), A\left(\xi, x_{2 n}(\xi)\right), t(\xi)\right), \\
M\left(A\left(\xi, x_{2 n+1}(\xi)\right), T\left(\xi, x_{2 n}(\xi)\right), t(\xi)\right), M\left(F\left(\xi, x_{2 n}(\xi)\right), A\left(\xi, x_{2 n}(\xi)\right), t(\xi)\right)
\end{array}\right\}\right]
$$$$
=r\left[\operatorname{Min}\left\{\begin{array}{l}
M\left(A\left(\xi, x_{2 n}(\xi)\right), A\left(\xi, x_{2 n-1}(\xi)\right), t(\xi)\right), M\left(A\left(\xi, x_{2 n}(\xi)\right), A\left(\xi, x_{2 n+1}(\xi)\right), t(\xi)\right), \\
M\left(A\left(\xi, x_{2 n}(\xi)\right), A\left(\xi, x_{2 n}(\xi)\right), t(\xi)\right), M\left(A\left(\xi, x_{2 n-1}(\xi)\right), A\left(\xi, x_{2 n}(\xi)\right), t(\xi)\right), \\
M\left(A\left(\xi, x_{2 n+1}(\xi)\right), A\left(\xi, x_{2 n-1}(\xi)\right), t(\xi)\right), M\left(A\left(\xi, x_{2 n-1}(\xi)\right), A\left(\xi, x_{2 n}(\xi)\right), t(\xi)\right)
\end{array}\right\}\right]
$$$$
\geq r\left[\operatorname{Min}\left\{\begin{array}{l}
M\left(A\left(\xi, x_{2 n}(\xi)\right), A\left(\xi, x_{2 n-1}(\xi)\right), t(\xi)\right), M\left(A\left(\xi, x_{2 n}(\xi)\right), A\left(\xi, x_{2 n+1}(\xi)\right), t(\xi)\right), \\
M\left(A\left(\xi, x_{2 n}(\xi)\right), A\left(\xi, x_{2 n}(\xi)\right), t(\xi)\right), M\left(A\left(\xi, x_{2 n-1}(\xi)\right), A\left(\xi, x_{2 n}(\xi)\right), t(\xi)\right), \\
M\left(A\left(\xi, x_{2 n+1}(\xi)\right), A\left(\xi, x_{2 n}(\xi)\right), t(\xi)\right), M\left(A\left(\xi, x_{2 n-1}(\xi)\right), A\left(\xi, x_{2 n}(\xi)\right), t(\xi)\right)
\end{array}\right]\right]
$$$$
=r\left[\operatorname{Min}\left\{G_{2 n-1}(\xi), G_{2 n}(\xi), 1, G_{2 n-1}(\xi), G_{2 n}(\xi), G_{2 n-1}(\xi), G_{2 n-1}(\xi)\right\}\right]
$$

If $G_{2 n-1}(\xi)>G_{2 n}(\xi)$, then $\left.G_{2 n} \xi\right)>r\left[G_{2 n-1}(\xi)\right]>G_{2 n-1}(\xi)$,

a contradiction therefore $G_{2 n-1}(\xi) \leq G_{2 n}(\xi)$

Therefore from $(1.3 .3), G_{2 n}(\xi) \geq r\left(G_{2 n-1}(\xi)\right) \geq G_{2 n-1}(\xi)$

Thus $\left\{G_{2 n}(\xi): n \geq 0\right\}$ is increasing sequence of positive real numbers in $[0,1]$ and therefore tends to a finite limit $\mathrm{L} \leqslant 1$.It is clear that $\mathrm{L}=1$ because if $\mathrm{L}<1$ then on taking Limit $\mathrm{n} \rightarrow \infty$ in (1.3.4) we get $\mathrm{L} \geqslant \mathrm{r}(\mathrm{L})>\mathrm{L}$, a contradiction. Hence $\mathrm{L}=1$ 
Now for any integer $m$,

$$
\begin{aligned}
& M\left(A\left(\xi, x_{n}(\xi)\right), A\left(\xi, x_{n+m}(\xi)\right), t(\xi)\right) \geq M\left(A\left(\xi, x_{n}(\xi)\right), A\left(\xi, x_{n+1}(\xi)\right), \frac{t(\xi)}{m}\right) *-- \\
& ---* M\left(A\left(\xi, x_{n+m-1}(\xi)\right), A\left(\xi, x_{n+m}(\xi)\right), \frac{t(\xi)}{m}\right) \\
& \geq M\left(A\left(\xi, x_{n}(\xi)\right), A\left(\xi, x_{n+1}(\xi)\right), \frac{t(\xi)}{m}\right) *-----* M\left(A\left(\xi, x_{n}(\xi)\right), A\left(\xi, x_{n+1}(\xi)\right), \frac{t(\xi)}{m}\right) \\
& \lim _{n \rightarrow \infty} M\left(A\left(\xi, x_{n}(\xi)\right), A\left(\xi, x_{n+1}(\xi)\right), t(\xi)\right) \geq 1 * 1 * 1 *-----* 1=1, \\
& \text { because } \lim _{n \rightarrow \infty} M\left(A\left(\xi, x_{n}(\xi)\right), A\left(\xi, x_{n+1}(\xi)\right), t(\xi)\right)=1 \text { for } t(\xi)>0
\end{aligned}
$$

Thus $\left\{\operatorname{Ax}_{\mathrm{n}}(\xi)\right\}$ is a Cauchy sequence and by the completeness of $\mathrm{X},\left\{\mathrm{Ax}_{\mathrm{n}}(\xi)\right\}$ converges to $\mathrm{u}(\xi) \in \mathrm{X}$. So subsequence $\left\{\mathrm{Fx}_{2 \mathrm{n}+1}(\xi)\right\}$ and $\left\{\operatorname{Tx}_{2 \mathrm{n}}(\xi)\right\}$ of $\left\{\mathrm{Ax}_{\mathrm{n}}(\xi)\right\}$ also converges to same point $\mathrm{u}(\xi)$. Since A is R-weakly commuting with F, so

$M\left(A\left(\xi, F\left(\xi, x_{2 n+1}(\xi)\right)\right), F\left(\xi, A\left(\xi, x_{2 n+1}(\xi)\right)\right), t(\xi)\right) \geq M\left(A\left(\xi, x_{2 n+1}(\xi)\right), F\left(\xi, x_{2 n+1}(\xi)\right), \frac{t(\xi)}{R}\right)$ On $\quad$ taking

$\lim _{n \rightarrow \infty}\left(\mathrm{A}\left(\xi, \mathrm{F}\left(\xi, \mathrm{x}_{2 \mathrm{n}+1}(\xi)\right)\right)=\lim _{n \rightarrow \infty} \mathrm{F}\left(\xi, \mathrm{A}\left(\xi, \mathrm{x}_{2 \mathrm{n}+1}(\xi)\right)\right)\right)=\mathrm{F}(\xi, \mathrm{u}(\xi)$.

Now we will prove that $\mathrm{F}(\xi, \mathrm{u}(\xi)=\mathrm{u}(\xi)$. First suppose that

$\mathrm{F}(\xi, \mathrm{u}(\xi) \neq \mathrm{u}(\xi)$ then there exists $\mathrm{t}(\xi)>0$ such that

$\mathrm{M}(\mathrm{F}(\xi, \mathrm{u}(\xi)), \mathrm{u}(\xi), \mathrm{t}(\xi))<1$

Now

$$
\begin{aligned}
& \left.\left.M\left(A\left(\xi, F\left(\xi, x_{2 n+1}(\xi)\right)\right), A\left(\xi, x_{2 n}(\xi)\right), t(\xi)\right) \geq r\left[M i n \begin{array}{l}
M\left(F\left(\xi, F\left(\xi, x_{2 n+1}(\xi)\right)\right), T\left(\xi, x_{2 n}(\xi)\right), t(\xi)\right), \\
M\left(F\left(\xi, F\left(\xi, x_{2 n+1}(\xi)\right)\right), A\left(\xi, F\left(\xi, x_{2 n+1}(\xi)\right), t(\xi)\right),\right. \\
M\left(F\left(\xi, F\left(\xi, x_{2 n+1}(\xi)\right)\right), A\left(\xi, x_{2 n}(\xi)\right), t(\xi)\right), \\
M\left(T\left(\xi, x_{2 n}(\xi)\right), A\left(\xi, x_{2 n}(\xi)\right), t(\xi)\right), \\
M\left(A\left(\xi, F\left(\xi, x_{2 n+1}(\xi)\right)\right), T\left(\xi, x_{2 n}(\xi), t(\xi)\right),\right. \\
M\left(F \left(\xi, x_{2 n}\left(, A x_{2 n}, t\right)\right.\right.
\end{array}\right\}\right)\right]
\end{aligned}
$$

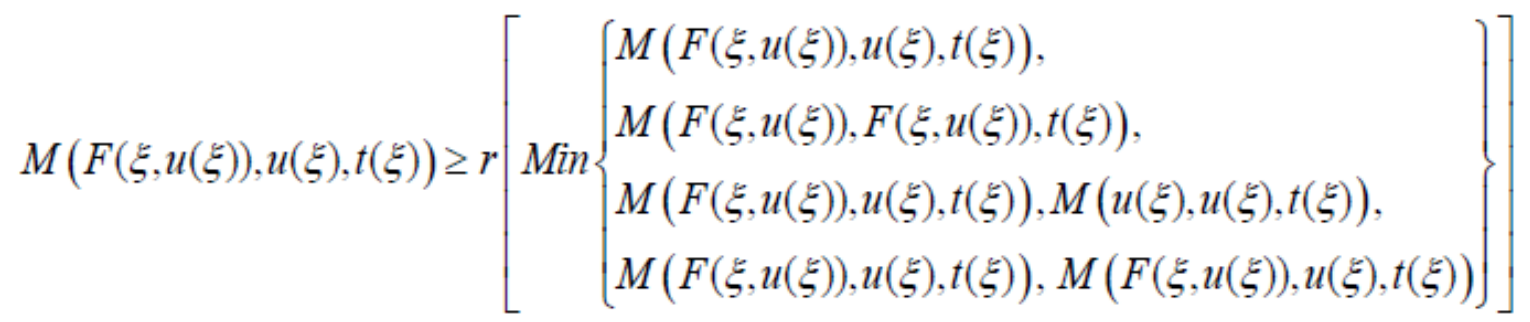

$$
\begin{aligned}
& M(F(\xi, u(\xi)), u(\xi), t(\xi)) \geq r[\{M(F(\xi, u(\xi)), u(\xi), t(\xi))\}]>M(F(\xi, u(\xi)), u(\xi), t(\xi)),
\end{aligned}
$$

which is a contradiction.

Thus $\mathrm{u}(\xi)$ is a fixed point of $\mathrm{F}$. similarly we can show that $\mathrm{u}$ is also fixed point of A. Now we claim that $u$ is fixed point of $T$. 
Suppose it is not so then for any $\mathrm{t}(\xi)>0, \mathrm{M}(\mathrm{u}(\xi, \mathrm{T}(\xi, \mathrm{u}(\xi), \mathrm{t}(\xi))<1$

$$
\begin{aligned}
& M\left(A \left(\xi, u(\xi), A\left(\xi, T\left(\xi, x_{2 n}(\xi), t(\xi)\right) \geq r\left[\operatorname{Min}\left\{\begin{array}{l}
M\left(F\left(\xi, u(\xi), T\left(\xi, T\left(\xi, x_{2 n}(\xi)\right)\right), t(\xi)\right),\right. \\
M(F(\xi, u(\xi)), A(\xi, u(\xi)), t(\xi)), \\
M\left(F(\xi, u(\xi)), A\left(\xi, T\left(\xi, x_{2 n}(\xi)\right)\right), t(\xi)\right), \\
M\left(T\left(\xi, T\left(\xi, x_{2 n}(\xi)\right)\right), A\left(\xi, T\left(\xi x_{2 n}(\xi)\right)\right), t(\xi)\right), \\
M\left(A(\xi, u(\xi)), T\left(\xi, T\left(\xi, x_{2 n}(\xi)\right)\right), t(\xi)\right), \\
M\left(F\left(\xi, T\left(\xi, x_{2 n}(\xi)\right)\right), A\left(\xi, T\left(\xi, x_{2 n}(\xi)\right)\right), t(\xi)\right)
\end{array}\right]\right)\right.\right.\right. \\
& M(u(\xi), T(\xi, u(\xi)), t(\xi)) \geq r\left[\operatorname{Min}\left\{\begin{array}{l}
M(u(\xi), T(\xi, u(\xi)), t(\xi)), M(u(\xi), u(\xi), t(\xi)), \\
M(u(\xi), T(\xi, u(\xi)), t(\xi)), M(T(\xi, u(\xi)), T(\xi, u(\xi)), t(\xi)), \\
M(u(\xi), T(\xi, u(\xi)), t(\xi)), M(T(\xi, u(\xi)), T(\xi, u(\xi)), t(\xi)))
\end{array}\right\}\right] \\
& M(u(\xi), T(\xi, u(\xi)), t(\xi)) \geq r[\operatorname{Min}\{M(u(\xi), T(\xi, u(\xi)), t(\xi))\}] \text {, } \\
& \text { which is contradiction. So } M(T(\xi, u(\xi)), u(\xi), t(\xi))=1
\end{aligned}
$$

Hence $u(\xi)$ is also a fixed point of T. That is $u(\xi)$ is common fixed point of T, F and A.

Uniqueness: Suppose there is another fixed point $\mathrm{v} \neq \mathrm{u}$, then

$$
\begin{aligned}
& M(A(\xi, u(\xi)), A(\xi, v(\xi)), t(\xi)) \geq r\left[\operatorname{Min}\left\{\begin{array}{l}
M(F(\xi, u(\xi)), T(\xi, v(\xi)), t(\xi)), \\
M(F(\xi, u(\xi)), A(\xi, u(\xi)), t(\xi)), \\
M(F(\xi, u(\xi)), A(\xi, v(\xi)), t(\xi)), \\
M(T(\xi, v(\xi)), A(\xi, v(\xi)), t(\xi)), \\
M(A(\xi, u(\xi)), T(\xi, v(\xi)), t(\xi)), \\
M(F(\xi, v(\xi)), A(\xi, v(\xi)), t(\xi))
\end{array}\right\}\right] \\
& M(u(\xi), v(\xi), t(\xi)) \geq r\left[\operatorname{Min}\left\{\begin{array}{l}
M(u(\xi), v(\xi), t(\xi)), M(u(\xi), u(\xi), t(\xi)), \\
M(u(\xi), v(\xi), t(\xi)), M(v(\xi), v(\xi), t(\xi)), \\
M(u(\xi), v(\xi), t(\xi)), M(v(\xi), v(\xi), t(\xi))
\end{array}\right\}\right] \\
& M(u(\xi), v(\xi), t(\xi)) \geq r[M(u(\xi), v(\xi), t(\xi))],
\end{aligned}
$$

which is a contradiction. So $\mathrm{u}(\xi)=\mathrm{v}(\xi)$.

Hence $\mathrm{A}, \mathrm{F}$ and $\mathrm{T}$ have unique common fixed point. 


\section{References}

[1] Beg, I. and Shahzad, N. "Random approximations and random fixed point theorems" J. Appl. Math. Stochastic Anal. 7 (1994). No 2, 145-150.

[2] Beg, I. and Shahzad, N. "Random fixed points of weakly inward operators in conical shells" J. Appl. Math, Stoch. Anal. 8(1995) 261-264.

[3] Bharucha -Reid, A.T. Fixed point theorems in probabilistic analysis, Bull. Amer. Math. Soc.82 (1976) 641-657.

[4] Choudhary, B.S. and Ray, M. "Convergence of an iteration leading to a solution of a random operator equation” J. Appl. Math. Stochastic Anal. 12 (1999). No 2,161-168.

[5] Choudhary, B.S. and Upadhyay, A. "An iteration leading to random solutions and fixed points of operators" Soochow J. Math.25 (1999). No 4,395-400.

[6] Choudhary, B.S. "A common unique fixed point theorem for two random operators in Hilbert spaces” I. J. M.M. S. 32 (2002) 177-182.

[7] Dhagat, V.B., Sharma, A. and Bhardwaj, R.K. "Fixed point theorem for random operators in Hilbert spaces" International Journal of Math. Analysis 2(2008) no. 12, 557-561.

[8] Grabiec, M.: Fixed points in fuzzy metric space, Fuzzy Sets and Systems, 27, 385- 389 (1988).

[9] Mishra, S.N., Sharma, N., Singh, S.L.: Common fixed points of maps on fuzzy metric spaces, Internet. J. Math. \& Math Sci., 17, 89-108 (1966).

[10] Som, T. "Some results on common fixed point in fuzzy Metric spaces" Soochow Journal of Mathematics 4 (2007) 553-561. 